\title{
Investigating Barriers for the Adoption of the German Contact-Tracing App and the Influence of a Video Intervention on User Acceptance
}

\author{
Victoria Böhm \\ University of Regensburg, media \\ informatics group \\ victoria.boehm@ur.de
}

\author{
Christian Wolff \\ University of Regensburg, media \\ informatics group \\ christian.wolff@ur.de
}

\author{
Corinna Geiselhart \\ University of Regensburg, media \\ informatics group \\ corinna.geiselhart@stud.uni- \\ regensburg.de
}

\author{
Eric Karl \\ University of Regensburg, media \\ informatics group \\ eric.karl@stud.uni-regensburg.de
}

\author{
Nina Kleindienst \\ University of Regensburg, media \\ informatics group \\ nina.kleindienst@stud.uni- \\ regensburg.de
}

\begin{abstract}
Despite the efforts that were put into data protection and data security, the overall adoption rate of the German contact-tracing app falls behind the estimated threshold of 60 percent which would be needed to suppress the virus effectively. Therefore, we conducted a questionnaire-based study to analyze barriers for the acceptance and to investigate the effect of a video intervention on the acceptance. Acceptance was measured using the technology acceptance model [15]. TAM measures were collected before and after watching the video intervention. Qualitative data on attitudes about the app was gathered through open questions and attitudinal items. 81 datasets from users with no prior experience were included in the further analysis. Results show that the video intervention did not increase behavioral intention (BI) significantly. However, the average scores of two important determinants of BI, Perceived Ease of Use (PEOU) and Perceived Usefulness (PU) increased significantly from 4.00 to 4.61 for PEOU $(\mathrm{p}<.00)$ and from 1.93 to 2.16 for PU $(\mathrm{p}<.001)$ with high and medium effect sizes. Qualitative data analysis indicates that the main barriers for adoption are perceived high risks and costs but low perceived personal benefits. One third of respondents (27/81) have privacy concerns and more than 40 percent of participants state they see "no benefits"(36/81).
\end{abstract}

\section{CCS CONCEPTS}

- Applied computing $\rightarrow$ Life and medical sciences; Health care information systems.

\section{KEYWORDS}

technology acceptance model, contact-tracing app, acceptance barriers

\footnotetext{
This work is licensed under a Creative Commons Attribution International 4.0 License.

MuC '21, September 05-08, 2021, Ingolstadt, Germany

(c) 2021 Copyright held by the owner/author(s).

ACM ISBN 978-1-4503-8645-6/21/09.

https://doi.org/10.1145/3473856.3474017
}

ACM Reference Format:

Victoria Böhm, Christian Wolff, Corinna Geiselhart, Eric Karl, and Nina Kleindienst. 2021. Investigating Barriers for the Adoption of the German Contact-Tracing App and the Influence of a Video Intervention on User Acceptance. In Mensch und Computer 2021 (MuC '21), September 05-08, 2021, Ingolstadt, Germany. ACM, New York, NY, USA, 8 pages. https://doi.org/10. $1145 / 3473856.3474017$

\section{INTRODUCTION}

To this date, 26.8 million people downloaded the German contacttracing app, roughly estimated one third of Germany's population [14]. Hinch et al. [6] published a mathematical model that proposes that the pandemic can be contained by an adoption rate of 56 percent of the population. This clearly leads to the question why users hesitate to install a warning app with prospects of such a promising benefit. Research must explore the possibilities and effectiveness of interventions to increase the adoption rates. The federal German government published an official information video about the German contact-tracing app, which describes key functionality and benefits without technical details so we wanted to investigate the effectiveness of this video as intervention for the adoption rate [4]. This study investigates three research questions: Can a video-based intervention (the official video on the CWA) increase the acceptance of users with no prior experience with the CWA? (RQ1). Does acceptance differ significantly across groups with different demographic characteristics, different attitude or information levels? (RQ2) What main reasons and concerns lead to the rejection of the CWA? (RQ3)

\section{RELATED WORK}

Two recent studies investigate the adoption rate and barriers for the German contact-tracing app. Both studies are highly relevant regarding our research questions but were not yet published at the time of experiment design or data collection. The questionnaire was online from August 20, 2020 to September 22, 2020. Blom et al. [2] predict the adoption rate based on data collected on three measures to model the adoption: willingness, accessibility and availability. They identify willingness as key barrier for the adoption, which 
underlines the necessity of investigating appropriate interventions to increase the willingness. In addition, they observed significant effects in the investigated subgroups of potential spreaders and vulnerable groups on access and ability. Further, they observed an age effect on access and ability. The older age groups are significantly less likely than younger age groups to use a compatible smartphone and to be able to install the app. Munzert et al. [13] combined a panel-based survey with tracking data to investigate uptake rates among demographic subgroups and to analyze the effectiveness video-based intervention and incentives as interventions. The results indicate that videos only have a small effect on uptake rates of the contact-tracing app, but small incentives can significantly increase the uptake rates.

From a methodological perspective, adoption or acceptance is operationalized very differently. Blom et al. [2] predict adoption based on three measures: access, ability and willingness. Munzert et al. use three measures: self reported uptake, tracked uptake as well as a combined metric [13]. Kahnbach et al. [8] treat download numbers as equivalent to adoption rate. Walrave et al. [16] investigated the factors that influence app use intention with a survey-based study using the extended unified theory of acceptance for modeling user acceptance (UTAUT).

Researchers identified several key factors and barriers for the acceptance of tracing apps. Zimmermann et al. [17] identified trust in authorities, respect of individual privacy, voluntariness, and temporary use of contact tracing apps as key factors for acceptance. Similar to the results of our qualitative data analysis, they observe the perception as surveillance tool. "[T]he concept of individual contact tracing was sometimes confused with population surveillance measures"[17]. Walrave et al. [16] found that the most important predictor was performance expectancy followed by facilitating conditions. Privacy concerns negatively influenced intention whereas participant's innovativeness was positively related with app use intention. Kahnbach et al. investigate quality characteristics of European contact tracing apps and associations with adoption rates [8]. Quality characteristics were measured using the German version of the Mobile App Rating Scale (MARS). Results suggest that that the quality characteristic, which seems to be most promising, is user engagement.

The idea to increase technology adoption by implementing appropriate interventions is already presented in 2008 by Venkatesh and Bala [15]. They describe the following seven categories of intervention: Design characteristics, user participation, management support, organizational support, incentive alignment, training and peer support.

Bedard et al. [1] investigate the effect of training on user acceptance of an electronic audit-workpaper system. Several other studies indicate that training is a good intervention to increase user acceptance $[7,11,12]$.

\section{METHOD}

\subsection{Procedure}

To gain insights about the effect of the official video on user acceptance, we designed a questionnaire based on a well-established framework for technology acceptance. The research instrument was distributed online and consisted of two main sections. The first part, called pre-questionnaire contains a brief introduction about the topic of the research instrument and a screening question for selecting appropriate participants. We asked participants, whether they had downloaded and used the CWA app. If they responded with yes, no further data were collected.

We recruited only participants with no prior experience to target more skeptical users but also to control for experience with the functionalities. The main part of the pre-questionnaire consisted of several items about the acceptance of the CWA based on the TAM model. Statements were taken from Claßen [3]. After filling out the pre-questionnaire, participants were asked to carefully watch the official video about the CWA, which was embedded in the questionnaire. The video published by the German Federal Government explains the main functionalities, data protection mechanisms and benefits, so we assumed it could positively affect acceptance in a similar way as training [4]. To check whether participants watched the video attentively and understood information given, several questions served as manipulation check. After the manipulation check questions, they were asked to rate the quality of the video. Then the same items as in the pre-questionnaire followed to measure the acceptance after watching the additional information.

\subsection{Measures}

We measured the attitude about Covid-19 with two statements concerning the emotions about being infected or infecting other people. Participants noted their level of agreement on a five-point Likert scale ranging from strongly agree $($ score $=5)$ to strongly disagree (score $=1)$.

For measuring the acceptance of the CWA, we used the items from Claßen [3] and adapted the statements by replacing the named technology. We chose to use these statements, because the questions are based on the original items from Venkatesh and Bala [15]. The questionnaire was the basis for our four main outcome variables for acceptance: perceived usefulness, perceived ease of use, current behavioral intention and future behavioral intention. To measure technology experience we used the items from the questionnaire from Karrer et al. [10]. The analysis from Claßen [3] and Kaspar [10] showed the instrument measures two dimensions, technology avoidance and technology interest. We assumed information behavior could influence the acceptance of the CWA, so we posed two open questions and two statements about research in the context of COVID-19. To address the research question why users hesitate to install and use the CWA, we formulated three open questions to measure the attitude towards the CWA (Why did not you install the Corona warning app? What advantages do you see from using the Corona warning app? What disadvantages do you see through the use the Corona warning app?). A factual manipulation check was implemented as defined by Kane and Barabas [9]. Participants were asked to rate six statements about information given in the video. Every participant with four or more correct answers was included. We assumed the quality of the content in the video would be essential for the video to be able to influence the perception of the CWA positively. So participants were asked to rate three key aspects of the video quality (communicating the app's functionality, its simplicity, and usefulness of the app). 


\subsection{Participants}

The questionnaire was deployed in several forums of our department, via Facebook and SurveyCircle. 122 respondents took part in the study, thirty-eight of whom were active users of the app and were excluded from the data collection. Three respondents failed in the manipulation test. This resulted in 81 valid data sets from 47 female participants, 33 male and one diverse respondent. The age of respondents ranged from 18 to 60 (Mean: 28.04, SD: 11.38).

\section{RESULTS}

\subsection{Evaluation of the video}

The average ratings show high agreement to all three statements, so we can assume that if the video does not change the judgement about the CWA, it is not because of the quality of the video. The mean ratings for the communication of benefits was 4.33 (SD 0.74) for communicating ease of use $4.11(\mathrm{SD}=0.91)$ and for the functionality 4.28 . $(\mathrm{SD}=0.85)$.

\subsection{Results RQ1: Pre and post-video acceptance}

To answer RQ1 if a video-based intervention (the official video on the CWA) can increase the acceptance of users with no prior experience with the CWA, we want to examine the following five hypotheses analogous to the work of Bedard (2003). Descriptions of all determinants of PU and PEOU are shown in Appendix A2. The video-based intervention is associated with increases in:

- H1: Perceived ease of Use (supported)

- H2: Perceived Usefulness (supported)

- H3: Behavioral intention (Present) (rejected)

- H4: Behavioral intention (Future) (rejected)

- H5: Computer Self Efficacy (supported)

For the analysis, pre- and post-training differences are tested by computing the average of individual measures within each construct, before and after watching the video and applying the related-sample Wilcoxon's Signed Rank test. The mean rating for Perceived Usefulness (PU) is 1.93 pre-video and 2.16 post-video $(\mathrm{z}=-3.38, \mathrm{p}<.001)$. In addition, the mean rating for Perceived Ease of Use (PEOU) is 4.00 in the pre-measurement and 4.61 in the postmeasurement $(\mathrm{z}=-5,398, \mathrm{p}<.000)$. The mean ratings for Present behavioral intention (BI) and Future behavioral intention (BIZ) did not differ significantly in pre and post-measurement. $\mathrm{H} 3$ and $\mathrm{H} 4$ are not supported. We observed three further significant upward shifts, in Computer Self Efficacy (CSE), Output Quality (OUT) and Result Demonstrability (RES). The mean rating of Computer Self Efficacy (CSE) was 2.70 in pre video measurement and 3.02 in the post-measurement $(\mathrm{z}=-2.826, \mathrm{p}<.005)$. So hypothesis 5 is also supported by the data. The Effect sizes for observed difference were medium for Perceived Usefulness, Computer Self Efficacy and Result demonstrability $(r>0.1)$ and high for Perceived Ease of Use and Output Quality $(r>5.0)$.

\subsection{Results RQ2: Group differences}

To investigate group differences, variables for demographic characteristics, attitudes, and information behavior were recoded into two different levels except education. Then descriptive statistics for behavioral intention to use (BI) were computed and tests for normality of the data. Results are summarized in Appendix A1. Mann-Whitney U tests were used to compare differences between the groups. For differences between groups of education levels, the Kruskall Wallis Test was used. Only statistically significant differences will be described in the following.

We found significant differences between groups with different anxiety-levels about an infection with COVID-19, different anxiety-levels about transmission of COVID-19, different levels of informedness about COVID-19 and informedness about the CWA. The mean rating for behavioral intention (BI) for users with higher levels of anxiety about a COVID 19 infection was 2.0 and for users with a lower level of anxiety $(1.0, \mathrm{U}=488.50, \mathrm{p}=.002)$. The effect size (Cohen, 1992) is medium, $r=0.33$. In addition the mean rating for BI of respondents with higher levels of anxiety about transmission of COVID-19 was 1.00 and for respondents with lower levels of anxiety about transmission of COVID-19 1.50 (U=515.00, $\mathrm{p}=.035)$. Effect size is medium, $r=0.245$. The Behavioral intention (BI) was greater for users with higher levels of informedness about COVID 19 with a mean rating of 1.5 than for lower levels with a mean of 1.0 $(\mathrm{U}=381.5, \mathrm{p}=.009 . \mathrm{r}=0.29)$. The mean rating of $\mathrm{BI}$ of participants with higher levels of informedness about the CWA is 2.0 and for users with lower levels of informedness $1.0(\mathrm{U}=586.000, \mathrm{p}=.022$, $\mathrm{r}=0.25$ ).

\subsection{Results RQ3: Attitude towards the contact-tracing app}

The answers to the three open questions were analyzed using an inductive content analysis approach as described by Elo and Kyngäs [5]. Every answer served as a primary unit for analysis. If one respondent gave several sentences with several reasons, every statement was coded separately. We started with specific categories and then defined more general codes. Specific and general codes were then translated into English. To examine the relative importance of answers, frequencies were computed for answer categories. Figure 1 and Figure 2 show answer categories and their frequencies. The qualitative findings point out two main reasons for the rejection of the CWA: The functionality is not perceived as useful and the app is connected with high costs like power consumption and perceived risks like for data privacy and mental stress.

Privacy concerns are the most frequently explained concern with 27 denominations among the disadvantages and 15 among reasons for not using the app as displayed in Figure 1 respectively Figure 2. In addition, the use of Bluetooth and the battery consumption seems to be an important issue with 15 respectively eight denominations. Although the app fulfills high standards for data privacy, the explained assumptions disclose a lack of knowledge about the implemented mechanisms for data protection and a lack of trust in the government.

Five times respondents used the term "surveillance" to express their feelings towards the app and another five times participants explicitly described the app would collect data about their location. Another disadvantage is the mental stress the app could cause. One respondent described he did not "want to live in fear".

Eight respondents mention a false sense of security, which could be caused and that users could start to act less cautious. 


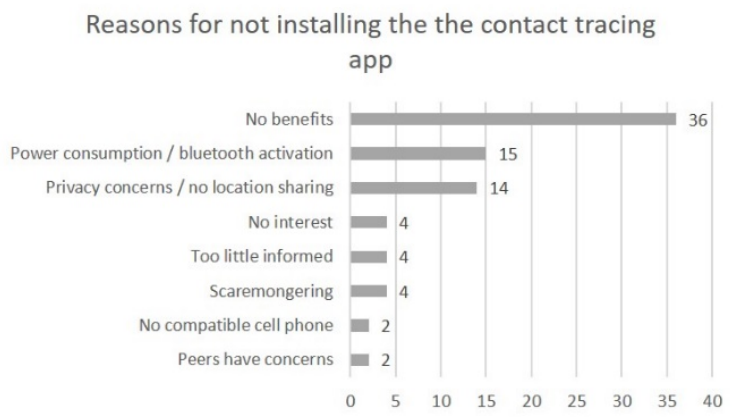

Figure 1: Reasons for not installing the app and disadvantages

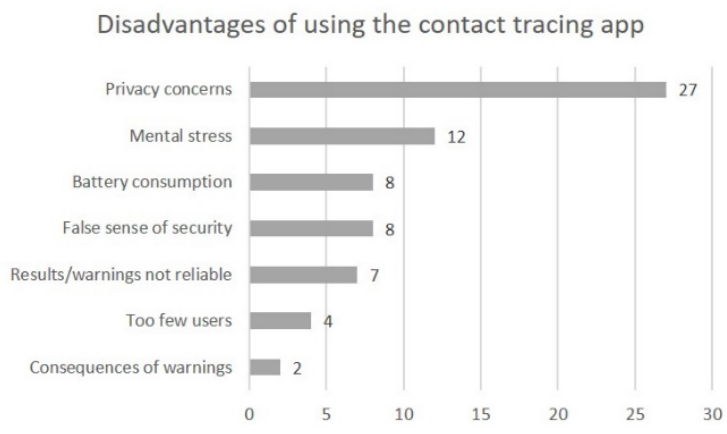

Figure 2: Disadvantages of using the contact-tracing app

Among the perceived advantages, the notification or warning was named by nearly 40 percent of all respondents (31/81). Fifteen persons claimed they do not see any benefits in using the CWA. The recognition and tracing of chains of infection was mentioned eleven times and the containment of the whole pandemic was named eight times.

The question about possible advantages reveals that users do acknowledge and know the main functionalities and benefits of the app (notification, chains of infection). Therefore, awareness about these two key functionalities might not be the reason for perceiving the app as "not useful". If we look at the specifications of advantages more in detail, the advantages are often described as "for others", e.g. elderly or vulnerable people. "People in the risk groups can be better protected". "It makes more sense for older people who are afraid of becoming infected " One explanation for the perception that they would not benefit from using the app might be the age of the sample, the mean age was 27 and the mean perceived threat of getting infected was rather low with 2.4. The threat of being a carrier was rated slightly higher with a mean of 3.06 Altogether; respondents know the theoretical advantages but perceive them as not very beneficial for them personally.

\section{CONCLUSION AND DISCUSSION}

In this study, we examine the ability of a video intervention to increase the user acceptance of the German contact-tracing app. We found that Behavioral Intention could not be significantly increased.
However, two important determinants for Behavioral Intention, Perceived Ease of Use and Perceived Usefulness increased with high and medium effect sizes. Qualitative data analysis of the open questions show that data privacy concerns and the perceived high costs (mental stress, battery consumption) together with the assumption that only the "others" would benefit from the app lead to the low willingness to install the CWA.

Our work complement the result from Blom et al. [2] and provide insights into reasons for hesitating to use the CWA of the less vulnerable groups. They found that less vulnerable groups are less willing to install the app and recommend targeting this user group. We advise to address the privacy concerns of these users and to include functionalities perceived as useful for the less vulnerable. In addition, our results point in the same direction as the work from Munzert et al., who showed that video interventions could not improve adoption significantly [13]. However, we operationalized adoption differently from others with using the TAM. Our findings show that videos can effectively communicate the usefulness and ease of use of the app, as these two determinants were significantly increased after the intervention. Therefore, videos could be used in future research to empirically investigate extended functionalities without implementation. Future work should further investigate the effectiveness of other interventions specifically targeted at less vulnerable users and elderly. In addition, long-term effects of interventions like incentives should be further investigated. Some limitations of the present study need to be acknowledged. We followed a repeated measures approach to detect pre and post-training differences. This could have affected the results.

\section{REFERENCES}

[1] Jean C. Bedard, Cynthia Jackson, Michael L. Ettredge, and Karla M. Johnstone. 2003. The effect of training on auditors' acceptance of an electronic work system. International fournal of Accounting Information Systems 4, 4: 227-250. https: //doi.org/10.1016/J.ACCINF.2003.05.001

[2] Annelies G. Blom, Alexander Wenz, Carina Cornesse, Tobias Rettig, Marina Fikel, Sabine Friedel, Katja Möhring, Elias Naumann, Maximiliane Reifenscheid, and Ulrich Krieger. 2021. Barriers to the large-scale adoption of a covid-19 contact tracing app in germany: Survey study. Fournal of Medical Internet Research 23, 3: e23362. https://doi.org/10.2196/23362

[3] Katrin Claßen. 2012. Zur Psychologie von Technikakzeptanz im höheren Lebensalter: Die Rolle von Technikgenerationen. Retrieved July 7, 2021 from http: //archiv.ub.uni-heidelberg.de/volltextserver/14295/

[4] Die Bundesregierung. 2020. Wie funktioniert und was kann die Corona-WarnApp. Retrieved July 11, 2020 from https://www.bundesregierung.de/breg-de/ themen/corona-warn-app/corona-warn-app-erklaerfilm-1758828

[5] Satu Elo and Helvi Kyngäs. 2008. The qualitative content analysis process. fournal of Advanced Nursing 62, 1: 107-115. https://doi.org/10.1111/J.1365-2648.2007. 04569.X

[6] Robert Hinch, Will Probert, Michelle Kendall, Chris Wymant, Matthew Hall, Katrina Lythgoe, Bulas Ana Cruz, Lele Zhao, Andrea Stewart, Luca Ferretti, Michael Parker, Ares Meroueh, Bryn Mathias, Scott Stevenson, Daneil Montero, Nicole K Mather, Anthony Finkelstein, Lucie Abeler-Domer, David Bonsall, and Christophe Fraser. 2020. Effective Configurations of a Digital Contact Tracing App: A report to NHSX. Retrieved July 7, 2021 from https://www.research.ox.ac.uk/Article/2020-04-16-digital-contact-tracing-canslow-or-even-stop-coronavirus-transmission-and-ease-us-out-of-lockdown

[7] Paul Jen Hwa Hu, Theodore H.K. Clark, and Will W. Ma. 2003. Examining technology acceptance by school teachers: A longitudinal study. Information and Management 41, 2: 227-241. https://doi.org/10.1016/S0378-7206(03)00050-8

[8] Leonie Kahnbach, Dirk Lehr, Jessica Brandenburger, Tim Mallwitz, Sophie Jent, Sandy Hannibal, Burkhardt Funk, and Monique Janneck. 2021. Quality and adoption of COVID-19 tracing apps and recommendations for development: Systematic interdisciplinary review of European apps. Fournal of Medical Internet Research 23, e27989. https://doi.org/10.2196/27989

[9] John V. Kane and Jason Barabas. 2019. No Harm in Checking: Using Factual Manipulation Checks to Assess Attentiveness in Experiments. American fournal 
of Political Science 63, 1: 234-249. https://doi.org/10.1111/AJPS.12396

[10] Roman Kaspar. 2003. Die Bedeutung der Technik für das Erleben von Einsamkeit im höheren Lebensalter. Retrieved July 7, 2021 from https://archiv.ub.uniheidelberg.de/volltextserver/5072/

[11] Marco Giovanni Mariani, Matteo Curcuruto, and Ivan Gaetani. 2013. Training opportunities, technology acceptance and job satisfaction: A study of Italian organizations. fournal of Workplace Learning 25, 7: 455-475. https://doi.org/10. 1108/JWL-12-2011-0071

[12] Bryan Marshall, Robert Mills, and David Olsen. 2008. The Role Of End-User Training In Technology Acceptance. Review of Business Information Systems (RBIS) 12, 2: 1-8. https://doi.org/10.19030/RBIS.V12I2.4384

[13] Simon Munzert, Peter Selb, Anita Gohdes, Lukas F. Stoetzer, and Will Lowe. 2021. Tracking and promoting the usage of a COVID-19 contact tracing app Nature Human Behaviour 2021 5:2 5, 2: 247-255. https://doi.org/10.1038/s41562020-01044-x

[14] Robert Koch Institut. 2021. KENNZAHLEN ZUR CORONA-WARN-APP DIGITALE ÜBERMITTLUNG VON TESTERGEBNISSEN 12,1 Mio. Retrieved
April 10, 2021 from https://www.rki.de/DE/Content/InfAZ/N/Neuartiges_ Coronavirus/WarnApp/Archiv_Kennzahlen/Kennzahlen_08042021.pdf?_blob=publicationFile

[15] Viswanath Venkatesh and Hillol Bala. 2008. Technology Acceptance Model 3 and a Research Agenda on Interventions. Decision Sciences 39, 2: 273-315. https: //doi.org/10.1111/j.1540-5915.2008.00192.x

[16] Michel Walrave, Cato Waeterloos, and Koen Ponnet. 2021. Ready or Not for Contact Tracing? Investigating the Adoption Intention of COVID-19 ContactTracing Technology Using an Extended Unified Theory of Acceptance and Use of Technology Model. Cyberpsychology, Behavior, and Social Networking 24, 6: 377-383. https://doi.org/10.1089/cyber.2020.0483

[17] Bettina Maria Zimmermann, Amelia Fiske, Barbara Prainsack, Nora Hangel, Stuart McLennan, and Alena Buyx. 2021. Early perceptions of COVID-19 contact tracing apps in German-speaking countries: Comparative mixed methods study. fournal of Medical Internet Research 23, 2. https://doi.org/10.2196/25525 
A APPENDICES

\section{A.1 Group Differences}

\begin{tabular}{|c|c|c|c|c|c|c|c|}
\hline Group & Mean BI & Median & $\mathrm{SD}$ & Lower bound & Upper bound & Kolmogorov & U Test \\
\hline $\begin{array}{l}\text { Age group1 } \\
(18-30)\end{array}$ & 1.91 & 1.50 & 1.07 & 1.64 & 2.19 & .000 & $\mathrm{Z}=-.548 ; \mathrm{p}=.584$ \\
\hline $\begin{array}{l}\text { Age group2 } \\
(31-60)\end{array}$ & 1.88 & 1.25 & 1.31 & 1.23 & 2.54 & .004 & \\
\hline Men & 1.96 & 1.50 & 1.19 & 1.54 & 2.39 & .000 & $\mathrm{z}=-0.362, \mathrm{p}=.716$ \\
\hline Women & 1.87 & 1.50 & 1.08 & 1.55 & 2.18 & .000 & \\
\hline Lower education & 1.50 & 1.00 & 1.00 & .45 & 2.54 & .016 & $\mathrm{H}=3.785, \mathrm{p}=.151$ \\
\hline Medium education & 1.81 & 1.50 & 1.04 & 1.5 & 2.10 & .000 & \\
\hline High education & 2.25 & 2.00 & 1.26 & 1.69 & 2.80 & .006 & \\
\hline Less vulnerable group & 1.919 & 1.50 & 1.08 & 1.65 & 2.18 & .00 & $\mathrm{z}=-.873, \mathrm{p}=.383$ \\
\hline Higher vuln. group & 1.84 & 1.0 & 1.32 & 1.043 & 2.64 & .00 & \\
\hline $\begin{array}{l}\text { No vulnerable person } \\
\text { in Household }\end{array}$ & 1.89 & 1.50 & 1.00 & 1.68 & 2.20 & .00 & $\mathrm{z}=0.375, \mathrm{p}=0.707$ \\
\hline $\begin{array}{l}\text { Household with } \\
\text { vulnerable Person }\end{array}$ & 1.92 & 1.50 & 1.24 & 1.51 & 2.330 & .00 & \\
\hline $\begin{array}{l}\text { Lower anxiety } \\
\text { infection }\end{array}$ & 1.57 & 1.00 & 0.83 & 1.32 & 1.81 & .000 & $\mathrm{z}=-3.045, \mathrm{p}=.002$ \\
\hline $\begin{array}{l}\text { Higher anxiety } \\
\text { infection }\end{array}$ & 2.39 & 2.00 & 1.29 & 1.93 & 2.85 & .008 & \\
\hline $\begin{array}{l}\text { Lower anxiety } \\
\text { transmission }\end{array}$ & 1.63 & 1.00 & 1.08 & 1.19 & 2.07 & .000 & $\mathrm{Z}=-2.112, \mathrm{p}=.035$ \\
\hline $\begin{array}{l}\text { Higher anxiety } \\
\text { transmission }\end{array}$ & 2.03 & 1.50 & 1.11 & 1.73 & 2.33 & .000 & \\
\hline $\begin{array}{l}\text { Less informed about } \\
\text { COVID }\end{array}$ & 1.37 & 1.00 & 0.62 & 1.08 & 1.66 & .000 & $\mathrm{Z}=-2.612, \mathrm{p}=.009$ \\
\hline $\begin{array}{l}\text { Higher informed } \\
\text { about COVID( }\end{array}$ & 2.08 & 1.50 & 1.18 & 1.77 & 2.38 & .000 & \\
\hline $\begin{array}{l}\text { Less informed about } \\
\text { CWA }\end{array}$ & 1.58 & 1.50 & 0.76 & 1.34 & 1.81 & .000 & $\mathrm{Z}=-2.282, \mathrm{p}=.022$ \\
\hline $\begin{array}{l}\text { Higher informed } \\
\text { about CWA }\end{array}$ & 2.27 & 2.00 & 1.32 & 1.83 & 2.71 & .001 & \\
\hline Less NPI Conformity & 1.62 & 1.25 & 0.79 & 0.96 & 2.28 & .054 & $Z=-.603, p=.546$ \\
\hline More NPI Conformity & 1.93 & 1.50 & 1.14 & 1.67 & 2.20 & .000 & \\
\hline Less Tech Devices & 1.95 & 1.50 & 1.18 & 1.45 & 2.45 & .001 & $\mathrm{Z}=-.022, \mathrm{p}=.983$ \\
\hline More Tech Devices & 1.88 & 1.50 & 1.09 & 1.59 & 2.17 & .000 & \\
\hline Tech interest low & 2.18 & 1.75 & 1.46 & 0.96 & 3.41 & .044 & $Z=-.264, p=.791$ \\
\hline Tech interest high & 1.87 & 1.50 & 1.07 & 1.62 & 2.12 & .000 & \\
\hline Tech Avoidance low & 1.93 & 1.50 & 1.10 & 1.66 & 2.20 & .000 & \\
\hline Tech avoidance high & 1.76 & 1.00 & 1.20 & 1.04 & 2.49 & .006 & $\mathrm{Z}=-.819, \mathrm{p}=.413$ \\
\hline
\end{tabular}




\section{A.2 DESCRIPTIVE STATISTICS AND RESULTS OF WILCOXON SIGNED RANK TESTS FOR PRE} AND POST-VIDEO MEASUREMENTS

\begin{tabular}{|c|c|c|c|c|c|c|c|c|}
\hline & Mean Pre & $\mathrm{SD}$ & Mean Post & $\mathrm{SD}$ & $\begin{array}{l}\text { Negative } \\
\text { Shifts }\end{array}$ & $\begin{array}{l}\text { Positive } \\
\text { Shifts }\end{array}$ & $\begin{array}{l}\text { Wilcoxon's } \\
\text { Signed Rank } \\
\text { Test (two tailed } \\
\text { p) }\end{array}$ & Effect Size \\
\hline PU & 1.93 & .99 & 2.16 & 1.07 & 14 & 33 & $-3.38(.001)$ & 0.375 \\
\hline PEOU & 4.00 & .794 & 4.61 & .67 & 6 & 45 & $-5.39(.000)$ & 0.599 \\
\hline $\mathrm{BI}$ & 1.90 & 1.11 & 2.01 & 1.22 & 13 & 21 & $-1.66(.097)$ & \\
\hline $\mathrm{BIZ}$ & 2.59 & 1.35 & 2.55 & 1.40 & 15 & 13 & $-.46(.642)$ & \\
\hline CSE & 2.70 & 1.51 & 3.02 & 1.70 & 12 & 28 & $-2.82(.005)$ & 0.314 \\
\hline PEC & 4.64 & .722 & 4.64 & .63 & 13 & 11 & $-.059(.953)$ & \\
\hline CANX & 2.31 & 1.12 & 2.18 & 1.07 & 31 & 20 & $-1.91(.056)$ & \\
\hline $\mathrm{ENJ}$ & 2.01 & 1.05 & 1.94 & .97 & 25 & 22 & $-.79(.429)$ & \\
\hline $\mathrm{SN}$ & 2.26 & 1.06 & 2.33 & 1.07 & 6 & 14 & $-1.27(.202)$ & \\
\hline REL & 1.72 & .880 & 1.72 & .95 & 16 & 18 & $-2.58(0.796)$ & \\
\hline OUT & 2.43 & 1.07 & 2.89 & 1.15 & 9 & 45 & $-4.65(.000)$ & 0.517 \\
\hline RES & 3.59 & .908 & 4.03 & .85 & 18 & 42 & $-3.49(.000)$ & 0.388 \\
\hline
\end{tabular}

\section{A.3 DETERMINANTS OF PERCEIVED USEFULNESS AND PERCEIVED EASE OF USE}

Determinants of PEOU (Venkatesh \& Bala, 2008)
Computer Self-Efficacy: The degree to which an individual
believes that he or she has the ability to perform a specific task/job
using the computer
Perception of External Control: The degree to which an
individual believes that organizational and technical resources exist
to support the use of the system
Computer Anxiety : The degree of "an individual's apprehension,
or even fear, when she/he is faced with the possibility of using
computers"
Computer Playfulness: The degree of cognitive spontaneity in
microcomputer interactions
Perceived Enjoyment The extent to which the activity of using a
specific system is perceived to be enjoyable in its own right, aside
from any performance consequences resulting from system use
Objective Usability: A comparison of systems based on the actual
level of effort required to completing specific tasks

Determinants of PU

Perceived Ease of Use The degree to which a person believes that using an IT will be free of effort

Subjective Norm: The degree to which an individual perceives that most people who are important to him think he should or should not use the system

Image: The degree to which an individual perceives that use of an innovation will enhance his or her status in his or her social system

Output Quality: The degree to which an individual believes that the system performs his or her job tasks well.

Job Relevance: The degree to which an individual believes that the target system is applicable to his or her job

Result Demonstrability: The degree to which an individual believes that the results of using a system are tangible, observable, and communicable

\section{A.4 ALTERNATIVE DESCRIPTION FOR FIGURE 1}

\begin{tabular}{ll}
\hline Categories & Frequencies \\
\hline No benefits & 36 \\
Power consumption & 15 \\
Privacy concerns & 14 \\
No Interest & 4 \\
Too little informed & 4 \\
Scaremongering & 4 \\
No compatible cell phone & 2 \\
Peers have concerns & 2 \\
\hline
\end{tabular}


A.5 ALTERNATIVE DESCRIPTION FOR FIGURE 2

\begin{tabular}{ll}
\hline Categories & Frequencies \\
\hline Privacy concerns & 27 \\
Mental stress & 12 \\
Battery consumption & 8 \\
False sense of security & 8 \\
Results/warning not reliable & 7 \\
Too few users & 2 \\
Consequences of warnings & 2 \\
\hline
\end{tabular}

